\title{
Relativistic Cowling approximation for fluid oscillation modes of color superconducting self-bound stars
}

\author{
German Lugones and Cesar Vasquez Flores \\ Universidade Federal do ABC, Brazil \\ email: german.lugones@ufabc.edu.br
}

\begin{abstract}
The investigation of the quasi-normal modes of oscillation of compact stars can reveal much information about their equation of state and internal structure mainly through the analysis of the expected emission of gravitational waves. In this work we study non-radial oscillation modes of strange stars consisting of color superconducting quark matter. We focus on the fundamental and pressure oscillation modes within the frame of the Cowling approximation. We discuss the observable features that may allow a differentiation among hadronic stars, strange stars, and strange stars with color superconductivity.
\end{abstract}

